\title{
Personalization Recommendation Framework in Mobile Computing
}

\author{
Wonseok kim \\ Dept. of Computer Engineering \\ University of Hannam \\ Daejeon, Republic of Korea \\ rntpwn5656@naver.com
}

\author{
Euiin Choi \\ Dept. of Computer Engineering \\ University of Hannam \\ Daejeon, Republic of Korea \\ eichoi@hnu.kr
}

\begin{abstract}
Recently, with the development of IT technology, the transition to the ubiquitous environment has been made so that various devices can be used to access information anytime and anywhere, and the computer can decide to provide useful services to the user. In this context aware mobile environment, context aware information is collected by sensors around the user and used to infer the user's current situation with personal information such as user profile. The inferred context information is used to provide a suitable service for the user. Recommendation techniques such as case based reasoning and collaborative filtering have also been studied in mobile computing, the web, and other fields. These technologies provide services by using personal profiles and user preference ratings. However, previous techniques used in ubiquitous computing did not take into account the privacy concerns and the accuracy of the contextual Therefore, access rights management is very important to adapt to information resources that requires the system. In this paper, we assume a context-aware model based on a profile of user information that provides efficient control rights to users through active classification, inference and resources. Therefore, a situation adaptive service recommendation technique is proposed in which location, time, and frequency information of frequently used services are stored, and services expected to be used at any location are stored in a storage device.
\end{abstract}

Keywords-Recommendation, Mobile, Personalization, LBS

\section{INTRODUCTION}

After the emergence of the ubiquitous environment, the user can connect to the computing environment each time using various devices, and the computer can decide to provide useful services to the user according to the context recognition. However, in this computing environment it is connected to wireless networks and various devices. The indiscriminate approach of information resources causes problems to the system. Therefore, access rights management is very important to adapt to information resources and systems by establishing a security policy that requires the system[2, 3, 4]. However, existing access control security model can access information resource or computing system using simple user ID and password. Therefore, there is a problem that this model is not related to the user context information. The access control model of the ubiquitous computing environment is different from the existing security access control model authorized by the user's simple information, and the user's location information, Device information can be checked in real time. It then provides services for user access requests through environmental information (location, time, etc.). If the authorized user, resource and authorized user access to the system is the same as the authorized user in order to use the service, then it is restricted to an authorized user who has authorization to access the requesting information. The access control model requires integration and automation of resource security by controlling according to user environment (same as context information) and user profile. In this paper, we propose a D-PACS (Dimension Profile and Access Control System) that stores location, time, and frequency information of frequently used services. In addition, there is a need for a personalization service framework that can be processed more quickly to provide a personalized service to a user that is suitable for retrieving the personalized service. We propose a context awareness framework that considers context information and profile of users who store personal information of each user, and environment setting $[1,5,6,7]$.

\section{RELATED WORKS}

\section{A. Cloud Computing}

Cloud computing is a very encouraging phenomenon As pointed out, there is little hope of getting a consensus or standard definition that is exactly the same as constructing a "cloud" (For example, the term grid is similarly overloaded), while emphasizing the quality of service contracts for the cloud and preparing for social issues with the technology infrastructure, while others are the price or nature of the resources provided (eq. storage, Processor, platform or application services). Some authors emphasize what the cloud offers to consumers, for example, services that are in demand. Others emphasize what is under the warehouse where the server is located. No single definition is "best" for all purposes. Fig. 1 shows the eight basic elements of cloud computing.

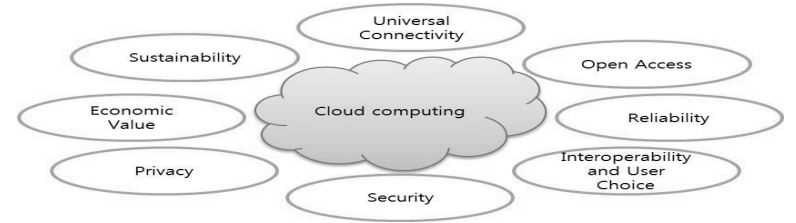

Fig. 1. Fundamental Elements of Cloud Computing 
- Service Platform (PaaS): It provides an existing managed and advanced software infrastructure for building specific classes of applications and services.

- The platform includes the use of underlying computing resources (generally billed similarly to IaaS products), but the infrastructure is abstracted under the platform.

- SaaS (Software as a Service): Provide a specific application that has already been created as a full or partial remote service. Sometimes it is in the form of a Web-based application and consists of standard non-remote applications with Internet-based storage or other network interactions.

\section{B. Collaborative Filtering}

Collaborative filtering is a technique for predicting users' interests using items obtained from many users, that is, preferences for products. It is mainly used for books, movies, VOD, music recommendation and so on. The preference for a product can be calculated based on the user's purchase history, view history, and rating information. The preference model should be changed in consideration of the industry domain and business characteristics of a company. Collaborative filtering is user-based and item-based as shown in [Fig. 2] and [Fig.3][8].

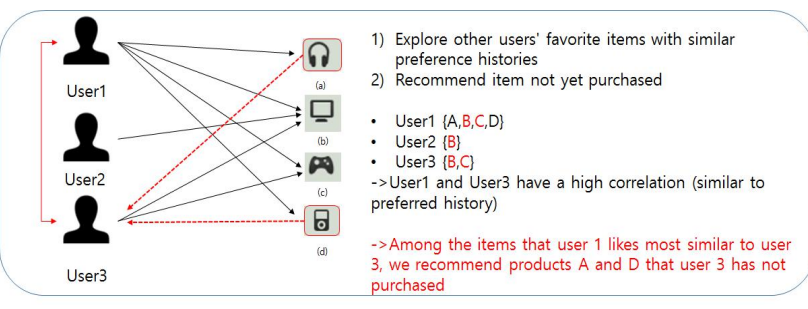

Fig. 2. User-Based

As shown in [Fig.3], 'User Collaborative Filtering' is a method that finds other users with similar preferences and bundles them into the same user group, and then recommends the products most preferred by other users in the same group.

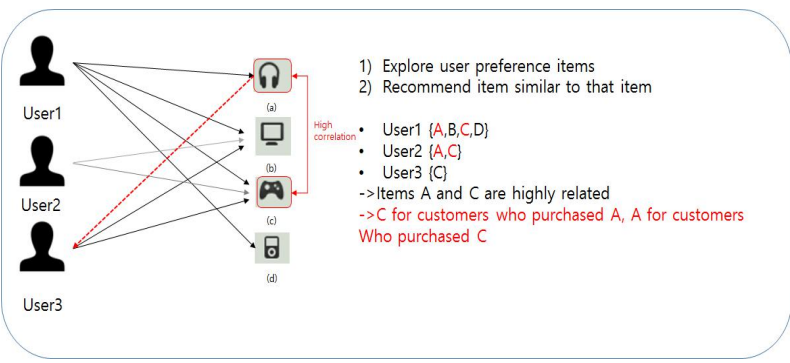

Fig. 3. Item-Based

As shown in [Fig.3], 'item-based collaborative filtering' is a method of recommending other items that are highly related to items based on user's favorite past purchase or inquiry items. Amazon also uses item-based collaboration filtering. Items that are similar to other users with similar preferences or that are related to past purchase items can be found through various distance calculation methods (Cosine, Euclidean, etc.) based on users' preferences for individual items. This is called

\section{FRAMEWORK}

In this paper, we propose a context awareness framework for personalized services in a mobile cloud computing environment that provides personalized services in a mobile cloud computing environment using frequently used service information and user information. Many existing frameworks for context awareness process location contexts rather than simply considering all the information in various contexts. Therefore, a context awareness framework is needed to provide faster and more efficient personalization services according to user environment. A context awareness framework for personalization services using mobile devices in mobile cloud computing has been proposed to provide uninterrupted service using user profiles including personal information and preferences[9]. The proposed framework is a mobile service cloud service provider that provides mobile agent, middleware and mobile cloud service that helps to use mobile cloud. Through the proposed framework of this paper, users can be provided with quick and continuous service when in a mobile state under any circumstance. Fig. 4 below shows the overall structure of the proposed personalization service framework.

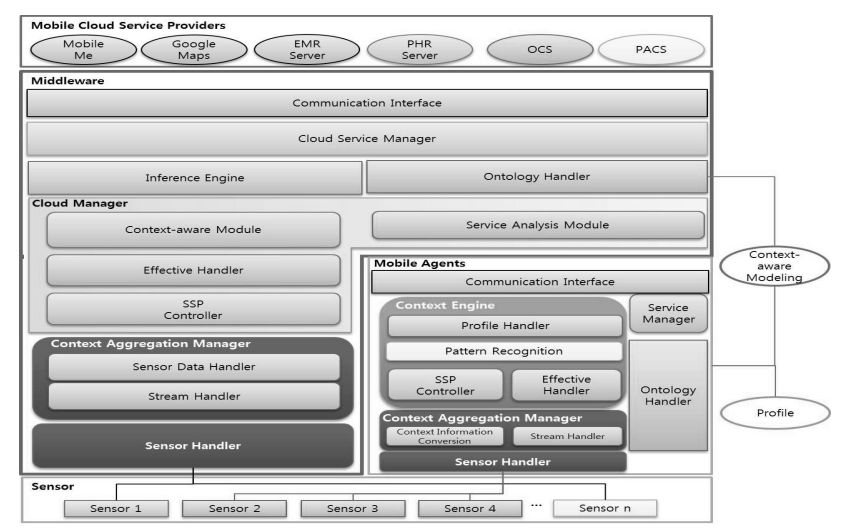

Fig. 4. The Whole Structure of Framework for Personalization Service

\section{A. Mobile Agent}

A mobile agent consists of a sensor handler, a context collection manager, a context engine, a service manager, an ontology handler, a profile, context aware modeling, and a communication interface. The sensor processor transfers the data stream transmitted from the various sensors to the processor. The context collection manager is data transmitted from the sensor to convert the digital stream processor and the converted digital data into the data type defined in the ontology composed of the sensor data. Context engine consisting of profile handler, pattern recognition, effective handler and sensor stream process controller. The profile processor processes the profile request and processes the pattern analysis. Pattern recognition is sensor data conversion data in a reception processor of an ontology that performs pattern analysis using rule and profile information. At this point, data redundancy is valid in the handler and must be able to request a scan. In addition, the Sensor Stream Processor Controller selects the sensor from which to collect data for that range. The profile stores the user's personal and preference information. Context 
aware modeling requires modeling for context information and context recognition. The service manager consists of the user interface and the service module, and the communication interface is the middleware and the mobile cloud service provider.

\section{B. D-PASC}

We need to deduce which service is suitable for the user to provide various services to the user-based contextual information generated in the ubiquitous environment. I usually stored my profile to use the user's propensity and information. Also, frequently used services have a high probability of continuing to use the services stored in the profile, thus reducing the use time. Therefore, a proposal has been proposed in which the information stored in the profile is frequently used and the time suggested. However, in order to provide a more accurate service, information on the user's location and time is required. For example, suppose an A service is used 10 times a day, and if it is 3:00 pm, it should be inferred that the service is used almost until afternoon. And the time and frequency of information are important in ubiquitous computing. However, location information is also very important. Although the services are the same, the frequency of services is different. Therefore, we propose a technique to provide the service requested by the user to store the location information with the time and frequency in the profile and to use the service in the time location. The proposed system consists of agents, DPACS, users, and service providers.

D-PACS consists of three modules: service manager, agent manager, inference engine, and each module consists of submodules. The Service Manager handles the services requested or expected by D-PACS. The predicted service is stored in the service storage. When a user requests an expected service from D-PACS, it searches directly from the service repository without a search operation. Therefore, the user can find the requested service more quickly and provide the service to the user. The agent manager receives the information from the DPACS manager at the agent and then sends it to the inference engine. Also, the service found by the agent is transferred from the service provider to the service handler. The analyzer in the inference engine analyzes the context with profile and sensor information to provide the appropriate service to the user and handle the access control of the user. The predictor then estimates the service the user will use the service elsewhere. In the inference engine, the authorization service module takes charge of management and processing in the context information of the subject and confirms the identity of the subject which can be accessed by the context aware access control system. In addition, the authorization service module provides a dynamic allocation service for the user role through the access policy analysis and acquires additional information such as access location, access time, and spatial domain to the context information of the subject, which is the resource The authentication service module performs access control role by comparing and analyzing security policies having both the currently active user role and the activated context role. The authentication service module is executed to monitor the access control function of the user. The authentication service module acquires the status information by the surround sensor or the device in addition to the access information of the accessed subject. And it is responsible for preprocessing the authority level of the user who wants to access by comparing and analyzing the situation information about the surround environment of the accessed user. And to provide data about the user's privileges through the authorization service. The contextual knowledge model database stores the contextual information of both the authentication service and the resource that wants to access the user. The user and role, constraint policy, contextual knowledge model represent information about the authorization, including access to the transaction list and requests for each transaction, as a type of rule indicating weakness or approval or approval. The context aware access control model uses Web Ontology Language (OWL) to collect and analyze context information about a user's surround environment.

\section{CONCLUSION}

Meaning of ubiquitous computing environment that can be conveniently and naturally used in computers in a common life without restriction of location or time. Thus, in a distributed computing environment such as a ubiquitous environment, a user is efficiently available and can share resources between users and other users. In the case of shared resources, it is also necessary to access a control model that can be accessed by an accessible user. And to use efficient resources that need access to a control model that can control unauthorized user access. Therefore, in this paper, the proposed model, D-PACS, will create an active access control system based on context awareness in ubiquitous environment. Assigns the appropriate role to the role of the information resource to which it is assigned and the role of access rights to the user. And provide the services available to the information resource through the appropriate access rights of the appropriate users. In addition, for active access control based on context awareness, it acts as a context through quantitative representation, which is the relationship between contextual information. In order to use the information resource, the context awareness-based active access control will be implemented as a validation evaluation on access control obtained by checking whether the security policy for the context role satisfies the security policy (despite the role assigned to the user). These changes in the computing environment increase the user's desire to provide differentiated services. Research on services is actively pursued in accordance with these requirements and most of these studies focus on providing personalized services provided by individual personality and context. It is very difficult to provide appropriate services to users in mobile environment because research on existing framework supporting personalization service only uses location information and other methods considering user's situation environment setting to provide appropriate service for user.

\section{ACKNOWLEDGMENT}

This work was supported by the Korea Sanhak Foundation (2017). 


\section{REFERENCES}

[1] Michael J. Covington, Matthew J. Moyer and Mustaque Ahamad, Generalized role-based access control for securing future application, NISSC, pp40-51, Oct 2000

[2] Michael J. Convington, Prahlad Fogla, Zhiyuan Zhan, and Mustaque Ahamad, Context-aware Security Architecture for Emerging Applications, Security Applications Conference(ACSAC), 2002.

[3] Gregory Biegel and Vinny Vahill, A Framework for Developing Mobile, Context-aware Applications, IEEE International Conference on Pervasive Computing and Communications(PerCom), 2004.

[4] T. Gu, H.K. Pung and D.Q. Zhang, A Middleware for Building ContextAware Mobile Services, In Proceedings of IEEE Vehicular Technology Conference(VTC), 2004.
[5] PARKHILL, D. The Challenge of the Computer Utility. AddisonWesley Educational Publishers Inc., US, 1966

[6] BECHTOLSHEIM, A. Cloud Computing and Cloud Networking. talk at UC Berkeley, December 2008.

[7] CHENG, D. PaaS-onomics: A CIO's Guide to using Platform-as-aService to Lower Costs of Application Initiatives While Improving the Business Value of IT. Tech. rep., LongJump, 2008.

[8] Changbok, Jang: Technique of Access Control and profile Storage on Ubiquitous Environment. ICHIT, 2010

[9] Bok-Man Jang: A Study of Context-Awareness RBAC Model using User Profile on Ubiquitous Computing. FGCN, 2010 